\title{
Exploración de estrategias de enseñanza orientadas a la inclusión de todos los alumnos en la clase de matemática. Resultados de un trabajo colaborativo entre docentes e investigadores
}

\author{
A search for teaching strategies aimed at the inclusion of \\ all students within the mathematical class. Results from a \\ collaborative work by teachers and researchers
}

\section{María Emilia Quaranta, ${ }^{1}$ María Mónica Becerril, ${ }^{2}$ Patricia García, ${ }^{3}$ María Paula Pérez, ${ }^{4}$ Patricia Sadovsky ${ }^{5}$}

\begin{abstract}
Resumen: Nuestra investigación se inscribe en una línea de trabajo en la que colaboramos con docentes de escuela primaria para analizar de manera conjunta problemas de enseñanza de la matemática. En una de las escuelas las maestras manifiestan preocupación porque en todos los grados existen niños que no logran participar del trabajo matemático con sus compañeros. Esta situación dio lugar a la realización de una pequeña indagación por parte de las mismas docentes para comprender mejor el posicionamiento de esos alumnos. El proceso de análisis que tuvo lugar a partir de la misma, así como la realización de una escritura conjunta permitieron en el grupo profundizar las relaciones entre enseñanza y aprendizaje, ajustar las interpretaciones que se hacían acerca de los conocimientos de los alumnos, subrayar el carácter institucional de los aprendizajes matemáticos de los niños y su inscripción en una trayectoria a lo largo del recorrido escolar.
\end{abstract}

Fecha de recepción: 21 de agosto de 2020. Fecha de aceptación: 03 de febrero de 2021.

\footnotetext{
Universidad Pedagógica Nacional, Argentina, memiliaquaranta@gmail.com. orcid.org/0000-0002-3760-2369 Universidad Pedagógica Nacional, Argentina, monicabece@gmail.com. orcid.org/0000-0002-3922-447X Universidad Pedagógica Nacional, Argentina, patgarcia41@speedy.com.ar. orcid.org/0000-0002-6247-0310 Universidad Pedagógica Nacional, Argentina, mpaulaperez48@gmail.com. orcid.org/0000-0001-9190-132X Universidad Pedagógica Nacional, Argentina, patsadov@gmail.com. orcid.org/0000-0001-6703-3315
} 
Palabras clave: investigación colaborativa - análisis de las prácticas docentes - enseñanza de la matemática - estrategias de intervención docente - inclusión de los alumnos.

\begin{abstract}
Our line of research involves collaborative work between researchers and primary school teachers in which we jointly analyze problems related to mathematics teaching. In one of the participating schools, the teachers express concern that in every grade there are children who are not able to engage in the mathematical work with their peers. This situation leads to an exploration by the teachers in order to better understand how these students are positioned. Both the analysis process as well as the collaborative writing process led the group develop a deeper understanding of the relationship between teaching and learning, an adjustment of the interpretations of students' knowledge, and an emphasis on the institutional nature of students' mathematical learning as part of their schooling trajectory.
\end{abstract}

Keywords: collaborative research - analysis of teaching practices - mathematics teaching and learning - teaching intervention strategies - inclusion of students.

\title{
1. INTRODUCCIÓN
}

Nuestro grupo de investigación ${ }^{6}$ estudia, desde una aproximación colaborativa (Bednarz, 2013), el funcionamiento de espacios de trabajo con maestros y directores de escuela primaria. Se busca analizar conjuntamente los problemas de enseñanza de la matemática que enfrentan las y los docentes en sus escuelas y explorar estrategias de intervención en las aulas que atiendan a las elaboraciones realizadas en el espacio colaborativo. ${ }^{7}$ La producción matemático-didáctica de los grupos en los que hemos participado y la problematicidad inherente al funcionamiento de la colaboración constituyen los ejes de la indagación (Sensevy, 2011).

\footnotetext{
6 Se enmarca institucionalmente en el departamento de Ciencias y Tecnología de la Universidad Pedagógica Nacional Unipe, Argentina.

7 En cada una de las escuelas en las que fuimos trabajando, hemos constituido un grupo con maestras y maestros que se integraban de manera voluntaria y al que también asistían integrantes del equipo directivo y con los que nos reuníamos quincenalmente. Denominamos espacios colaborativos (EC) a estos ámbitos de trabajo.
} 
Diversos artículos comunican resultados que fuimos obteniendo. Nos hemos referido a los modos en que los intercambios han dado lugar a la identificación de nuevos contenidos a enseñar (Sadovsky et al., 2015) y al papel de la interpretación compartida de las estrategias de los alumnos para alcanzar una mayor comprensión de la enseñanza de la matemática (Sadovsky et al., 2015). Nos hemos referido también a la identificación, en el marco de las discusiones con las maestras, de ejes de análisis para la clase (Sadovsky et al., 2019) y a problemas metodológicos inherentes a la consolidación de un intercambio colaborativo, asumida una perspectiva en la que buscamos que las distintas posiciones se puedan expresar de manera fundamentada en un clima de confianza (Sadovsky et al., 2016; Sadovsky et al., 2019).

En la Escuela 30, una de las instituciones en las que veníamos trabajando desde el año 2016 con las maestras de $1^{\circ}$ a $4^{\circ}$ grado y su directora, la intervención docente con relación a los aprendizajes de los alumnos -su identificación, la diversidad entre los niños, los progresos individuales y colectivos, el grado de estabilidad alcanzada, los modos de ayudar- fue un eje organizador de las conversaciones del grupo a partir del cual se derivaron distintas dimensiones de análisis (Sadovsky et al., 2019). Una de ellas, en particular, refiere a una tensión permanente por parte de las maestras entre guiar a los alumnos hacia la respuesta esperada y promover el desarrollo de sus ideas como estructurante de la interacción. Es decir, frente al desconcierto que generan ciertas producciones de los niños, se reconoce que muy frecuentemente se opta por ir comunicando el modo más convencional de resolución.

En el inicio de las reuniones de 2018, las docentes visualizan un panorama preocupante: por una parte, llegan varios chicos a primer grado sin haber asistido al nivel inicial y, en consecuencia, ajenos a algunas prácticas escolares que las maestras suelen considerar disponibles; por otra, alumnos de diferentes grados -no se sabe precisar cuántos- no llegan a involucrarse en las propuestas de trabajo matemático, fundamentalmente en la resolución de problemas aritméticos.

La directora de la escuela plantea con fuerza que, este problema es institucional y requiere encontrar un modo de abordarlo. Subraya la necesidad de interactuar con aquellos niños que parecen distantes frente a las tareas como condición para comprender cómo están pensando e intervenir para que vayan 
cambiando de posición. Los registros de las reuniones ${ }^{8}$ muestran que retoma en varias oportunidades la dualidad ya discutida entre guiar a los alumnos y promover el desarrollo de sus ideas para dar marco a la elaboración que propone. Al mismo tiempo, señala la complejidad que esto supone y valoriza el EC como una oportunidad para construir estrategias de manera conjunta. Asume una posición inclusiva que marca con contundencia una orientación pedagógica central y reconoce que para poder sostenerla se requieren prácticas específicas que es necesario producir. Releva, asimismo, que producir estrategias que contribuyan a que todos los alumnos se involucren en el trabajo matemático no se reduce a una cuestión de voluntad. Nos interesa resaltar el papel que cumple el EC en hacer visible para ella -y para todas- el componente de producción original que supone la gestión de las orientaciones pedagógicas desde el papel del directivo.

La problemática que presenta la directora es compartida, de distintas maneras, por el grupo de docentes. La maestra de tercer grado agrega dos cuestiones estrechamente relacionadas, referidas al progreso de los alumnos. Expone su preocupación por lograr que los niños avancen en las estrategias de cálculo ya que, según su visión, la mayoría apelaba a recursos de conteo. Esto la lleva a expresar el interés de realizar acuerdos en todo el equipo docente respecto de los procedimientos que se enseñarían en cada grado. Analiza también que, cuando intenta explicar para todos nuevos procedimientos más elaborados, algunos alumnos quedan sin comprender. Entendemos que en este planteo está implícita otra dualidad ya tratada en el EC: la relación entre las interacciones personales y las grupales en el aula (Sadovsky et al., 2019). Notemos que esta docente amplía el problema originalmente planteado por la directora: es necesario mirar la inclusión de los niños en la tarea matemática -también- considerando los chicos que no comprenden las explicaciones grupales.

En este contexto, y como estrategia para abordar las cuestiones expuestas, la maestra de cuarto grado propone un proyecto colectivo: plantear a todos los alumnos - de primero a sexto- un problema que implicara una resta, un enunciado con la misma estructura para toda la escuela, adaptando los datos a cada grado. Más específicamente, proyecta un dispositivo en dos etapas: en la

\footnotetext{
8 Como indicamos en otros trabajos en los que nos detuvimos en las estrategias metodológicas de la investigación, todas las reuniones son grabadas. Entre cada encuentro y el siguiente, se elaboran registros integrando la desgrabación y las notas de las investigadoras, se realiza un primer análisis para preparar una síntesis escrita de la reunión, con las principales cuestiones discutidas, así como también interpretaciones del equipo de investigación que proponemos como hipótesis a validar/confrontar con las docentes. Estas síntesis constituyen una referencia para el inicio de la reunión siguiente y el avance de las conversaciones.
} 
primera, los alumnos resolverían sin ayuda -salvo las aclaraciones relativas a una primera comprensión de la tarea- y, en la segunda, que se realizaría en los días siguientes, cada docente interactuaría con los chicos que, por diferentes razones, no pudieron avanzar en una resolución. Del desarrollo de esta propuesta - de las discusiones a las que dio lugar, de las transformaciones con relación al sentido que el grupo le fue atribuyendo a este accionar, del papel que jugó la escritura colectiva de los propósitos- damos cuenta a continuación.

\section{RELEVAR LAS PRODUCCIONES DE LOS ALUMNOS PARA REPENSAR LA INCLUSIÓN DE TODOS EN EL TRABAJO MATEMÁTICO ESCOLAR}

La maestra de cuarto grado concibe el dispositivo que sugiere como respuesta a los problemas que se estaban discutiendo en el grupo colaborativo. En primer lugar, como una oportunidad para disponer de producciones de todos los alumnos para analizar en el EC. El análisis de trabajos escritos de los niños era una práctica ya instalada desde el primer año de funcionamiento. En esta ocasión se busca generar un conjunto de datos para explorar según las preocupaciones compartidas. Esta docente anticipa que las producciones pondrán de manifiesto distintos tipos de dificultades. Señala también que esta exploración va a constituir una oportunidad para vincular la interpretación de las producciones de los alumnos, sobre todo de algunos de ellos, en términos de ideas y dificultades respecto del trabajo matemático, con posibles intervenciones docentes que los ayudarían a progresar. Explicita su intención al diferenciar los dos momentos de la indagación que mencionamos: a) confrontar aquello que los niños pueden sin y con ayuda y hacer visible de esta manera el papel de la intervención; b) elaborar posibles mediaciones para esas situaciones. Qué les pasa a aquellos alumnos que parecen tan distantes cuando enfrentan la tarea y cómo los ayudamos, parecen ser preguntas que subyacen a la propuesta:

M $4^{09}$ : Haríamos un primer trabajo para ver qué hacen y un trabajo posterior a partir de eso. No es solamente hacer un diagnóstico sino, después, volviendo, viendo las realidades ...

9 Maestra de $4^{\circ}$ grado. 
$\mathrm{D}^{10}$ : Claro, lo que decís, qué pasa sin ninguna intervención y después qué pasa con las intervenciones.

M $4^{\circ}$ : Cómo intervenimos en esos casos.

D: Esas hojas en blanco, cómo se interviene.

M $4^{\circ}$ : Y si aparece algo que vos decís "y esto no tiene nada que ver", ver si aparece en uno solo, si aparece en varios y tratar de entender por qué aparece eso.

Se va haciendo explícito el sentido de los dos momentos que concibe para la indagación y el tipo de análisis que se imagina, señala la complejidad que tiene la interpretación de algunas producciones, expresa la necesidad de pensar en las intervenciones en el largo plazo y de coordinar las trayectorias personales de los niños con el avance de cada grupo:

M $4^{\circ}$ : En un segundo paso, qué hacemos con los resultados que tenemos. A partir de los resultados, vamos a tener que ver si un gran porcentaje no hace o un gran porcentaje hace cosas que... Supongamos que algunas nos cuesta entender pero después uno termina entendiendo por qué hacen lo que hacen... Chicas, todas tenemos nenes que quizás uno les da una situación y hacen cosas que realmente, ni ellos pueden explicarlas (...) donde uno tiene que intervenir un poco más, tiene que guiar un poco más. Digo, primero saber cuál es nuestra realidad y, entonces, una vez que sabemos la realidad, me parece que vamos a poder determinar qué hacer con nuestros alumnos y después acordarnos, cómo vamos a continuar con ellos el año que viene.

A medida que se van precisando y compartiendo en el EC las preocupaciones de algunas maestras relativas a la inclusión y al avance de todos los niños, cobra forma un dispositivo en el que se convoca al colectivo docente a embarcarse en la búsqueda de datos cuyo análisis permitiría avanzar en la elaboración de estrategias para la acción docente como respuestas a los problemas compartidos. Notemos la aproximación investigativa de esta propuesta, novedosa como iniciativa, que surge desde el interior de las prácticas docentes y, entendemos, fue favorecida por el desarrollo del grupo colaborativo.

10 Directora 


\section{LOS INTERCAMBIOS A RAÍZ DE LAS PRODUCCIONES RECOLECTADAS: UN ESPACIO PARA TRANSFORMAR SENTIDOS, EVOCAR EXPERIENCIAS Y FORMULAR PROPUESTAS}

Cuando las docentes traen al EC las producciones recolectadas en sus respectivas aulas y se comparte una primera ronda de relatos, se inicia un proceso de análisis que va enriqueciendo el sentido original de la indagación. A raíz de las resoluciones de los niños obtenidas en cada grado, se evocan en la conversación experiencias ya realizadas, se hacen interpretaciones posibles y se formulan nuevas propuestas que se entraman con la reflexión compartida sobre el material. Al disponer de un conjunto de datos recogidos con un propósito específico, se hace posible tomar distancia de las acciones de enseñanza más inmediatas que solían ocupar los intercambios del grupo y se favorece el tratamiento de cuestiones que atraviesan las prácticas de toda la institución, aun con las distintas interpretaciones de cada integrante. La consideración de las producciones de los niños habilita una reflexión que se refiere simultáneamente al alumno, a su grado y a la escuela. Asimismo, fue notorio, en el momento de presentación y análisis de los datos, que cada maestra orientó su mirada en función de las preocupaciones que había manifestado cuando se preparaba esta indagación.

En el proceso de análisis que tuvo lugar en el EC a raíz del dispositivo de indagación, los aportes de las maestras y de la directora, en algunos momentos, refieren al valor de la intervención docente y a posibles estrategias de acción de cara a los asuntos que preocupan y, en otros, se centran más en los recursos que necesitan para interpretar las ideas que pusieron en juego los alumnos, desde una posición en la que la intención de comprender posterga momentáneamente las urgencias de la acción. Es claro que estas dos perspectivas, cuyos aspectos principales desarrollaremos a continuación, se entraman permanentemente en la conversación y las separamos solo para organizar la comunicación.

\subsection{ESTRATEGIAS DE ACCIÓN}

En un primer análisis, cada maestra va desplegando las estrategias puestas en juego por sus alumnos y sus compañeras van realizando aportes. La directora propone hacer foco en aquellos niños que entregan su hoja en blanco. ¿Cómo hacer un análisis sobre la base de una hoja en blanco? La convocatoria es intencional y llama a la necesidad de hacerse preguntas que remiten tanto a la 
tarea propuesta como al recorrido de los niños en su relación con el trabajo matemático del aula, más allá de una producción puntual. En ese contexto, se analiza el caso de primer grado que, como dijimos, venía preocupando al grupo por el ingreso de muchos niños que no habían asistido al nivel inicial.

El problema propuesto a los niños, que se mostró desajustado solo para unos pocos, plantea la complejidad inherente a la necesidad de interactuar con todos los alumnos en un aula, siempre diversa. La maestra correspondiente pide ayuda a las integrantes del EC (Por favor, chicas, díganme, qué hacer), a raíz de lo cual se despliegan algunas ideas para explorar en las aulas. La maestra de cuarto grado, a través de algunas sugerencias, trae un tema nodal, sobre todo en el momento de entrada de los alumnos en el trabajo matemático: la relación entre prácticas sociales que pueden constituirse en referencias y su matematización. Efectivamente, a raíz del enunciado planteado por la maestra de primero -Yamila compró 12 figuritas pero 4 eran repetidas, ¿cuántas pudo pegar en el álbum?-, expresa:

M $4^{\circ}$ : Si vos traés las figuritas y hacés como un álbum, proponerle al chico que lo haga, y están las repetidas y, contamos cuántas se pudieron pegar... Como un primer paso, obviamente que uno después trata..., pero quizás llevarlo a instancias de ese estilo.

M $1^{\circ}$ : Eso está bueno, eso vamos a poder hacerlo.

Otras maestras sugieren: contar el material que se reparte en el aula, seleccionar una cierta cantidad de elementos para que cada niño tenga uno. Todas ellas apuntan tanto a ampliar el caudal de experiencias de los niños como a subrayar que muchas de las acciones que se realizan en la vida cotidiana de la clase admiten una interpretación en términos matemáticos. De esta manera, emerge una cuestión de interés en las discusiones con los maestros: la necesidad de tomar en cuenta una operación intelectual que ineludiblemente deben realizar los alumnos en un proceso de matematización, la de recortar los datos relevantes de la propuesta que se les hace, operar con ellos y responder las preguntas planteadas (Peltier-Barbier, 2004). Como sabemos, este proceso requiere comprender la situación más amplia desde la cual se abstrae el enunciado. Entendemos que la sugerencia de las maestras habilita a tematizar esta cuestión en el grupo y superar las interpretaciones frecuentes que reducen la ausencia de respuestas a una dificultad en la comprensión de la consigna. En esta conversación, se hace explícita también la diferencia entre apelar a una escenificación de la situación referida y a representaciones gráficas diversas que, si bien pueden 
concebirse como mediadoras en algún momento en el proceso de aprendizaje, no portan la misma carga de significación cuando la experiencia y su función como referente están ausentes.

Inscribimos en la misma tematización otro aporte de la maestra de cuarto grado, esta vez orientado a los niños que operan con los datos del problema pero, una vez centrados en el trabajo numérico, parecen perder de vista la situación de partida, como si la realización de cálculos en sí misma fuera lo más relevante del trabajo matemático. Ella propone pedir a los alumnos que expliciten la respuesta como un modo de favorecer ese retorno (Es esto de no perder a dónde íbamos). La discusión generada a propósito de esta última cuestión permite resignificar una antigua práctica -la de escribir la respuesta de los problemas- que tenía históricamente un carácter formal y muchas veces desprovisto de sentido para los niños. Por el contrario, en este caso se trataría de enfatizar, a través de intervenciones específicas, los vínculos entre la situación cotidiana de referencia, el problema matemático que de ella se recorta, el tratamiento numérico que admite y la respuesta obtenida. Cabe señalar que esta maestra había traído en reiteradas oportunidades a la mesa de trabajo ejemplos de su aula que daban cuenta de la necesidad de trabajar diferentes aspectos de las relaciones recién mencionadas.

La hoja en blanco convoca también a detenerse en el recorrido matemático de los niños. Al respecto, la directora insiste en ir a buscar las ideas de los alumnos, no las que esperamos, como condición ineludible para entablar una genuina interacción. Ella subraya que muchas veces, frente a respuestas desconcertantes, los maestros tienden a pensar que los chicos no saben nada y, al no encontrar modos de interactuar con ellos, terminan excluyéndolos, aun cuando sean docentes con una intención inclusiva y que alientan la diversidad de producciones en el aula. Interpretamos que todo ocurre como si los docentes funcionaran en sus interacciones en la clase esperando un espectro de intervenciones posibles por parte de los niños, fuera del cual les resulta muy difícil retomar su pensamiento para contribuir a transformarlo. Justamente el EC -esta es una hipótesis que surge de nuestro estudio- cumpliría la función de ampliar ese espectro, cuya mayor o menor flexibilidad podríamos pensarla dependiente de una posición que se mueve entre dos polos en tensión: uno más abierto y centrado en la búsqueda de las ideas de los niños, otro más cerrado e inclinado hacia la búsqueda de concordancia con un conjunto de expectativas.

Entendemos que la posición que se asuma frente a esta dualidad entre ir a buscar a los niños o esperar unas respuestas anticipadas depende de la 
convicción de que vale la pena atribuir -a título hipotético- estatuto de conocimiento a las ideas de los niños y de la confianza en la fertilidad de una interacción con ellas (Robert, 2003). Compartir los análisis acerca de los intercambios en la clase, elaborar hipótesis al respecto y explorarlas en la práctica son componentes de la construcción de ese posicionamiento.

En esta dirección interpretamos las intervenciones de la directora en el EC cuando insiste en la necesidad de entender lo que hacen los chicos y apelar a la colaboración en el equipo cuando se hace difícil esa comprensión:

D: No siempre es fácil saber qué está pensando el chico porque a veces tampoco ellos lo pueden verbalizar, y eso me parece que es el trabajo más valioso que hacen ustedes, ese poder intervenir y buscar en cada uno cómo ayudarlos a ir avanzando. La intervención docente es difícil, por eso estos espacios son ideales para esto. Algunos chicos aprenden a pesar de todo y otros, si no hay intervención docente, no aprenden.

A lo largo de las reuniones, son numerosas las expresiones de este tipo por parte de la directora en las que subraya la necesidad y la complejidad de la intervención docente, el costo afectivo en términos de frustración que muchas veces tiene la dificultad para comprender lo que los niños hacen. Agrega que estas intervenciones requieren elaboraciones específicas en función del contenido tratado. En este contexto en el que se instala la idea de ir a buscar a los niños, apela a un ejemplo de la propia escuela, que refiere a un alumno de tercer grado de los que dejaban la hoja en blanco y que, a través de un proceso de interacciones sostenidas con él a lo largo de los años, logra avances significativos. Para ilustrarlos, trae a la mesa una resolución reciente del niño, a raíz de un problema parecido a los de la indagación (En un salón hay 93 sillas y 18 personas sentadas. ¿Cuántas sillas vacías hay?). [figura 1] 


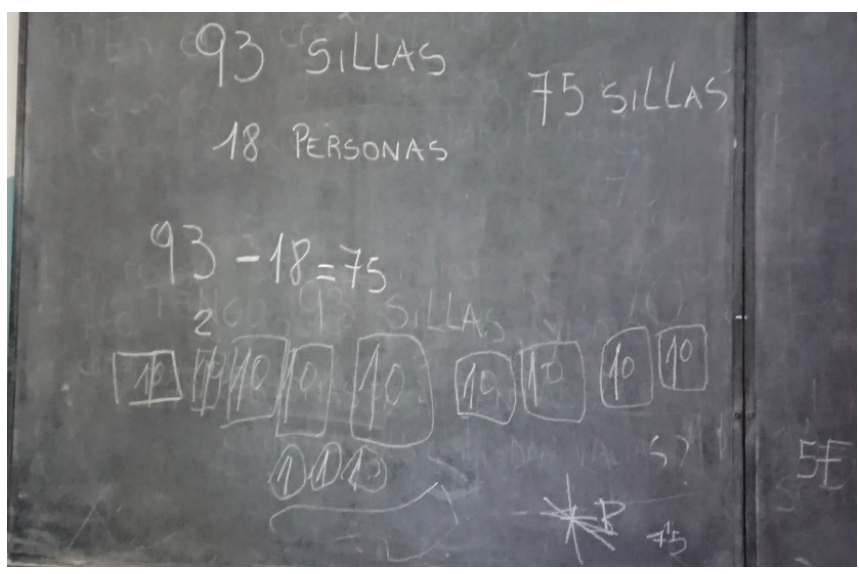

Figura 1. Producción de un alumno que logra avances significativos a partir de un proceso de interacciones sostenidas.

Este niño reconoce la resta como una operación que le permite encontrar la diferencia solicitada y apela a una descomposición de los números para operar. Pero, recién cuando explica que el 2 -que está arriba del 10 tachado- es como dar el vuelto, la directora puede interpretar que los cuadraditos dibujados representan para él billetes. Es decir, implícitamente apela a otro marco -el contexto del dinero- para representar y resolver la situación (Douady, 1984). Notable operación intelectual para un niño que, en el inicio de su escolaridad, como dijimos, dejaba la hoja en blanco y que, cuando se puso foco en él y las docentes se sentaban a su lado acompañándolo en sus resoluciones, podía poner de manifiesto su comprensión de los problemas.

En el marco de análisis generado en el EC a raíz de esta indagación, cobran nueva significación experiencias acumuladas en la institución de las cuales se extraen elementos para enriquecer las elaboraciones del grupo a raíz de los problemas que se están tratando. En este sentido, toda la construcción del grupo actúa como marco de análisis y los casos referidos exceden completamente el material recolectado para esta ocasión.

El problema de los niños que quedan afuera fue analizado hasta acá considerando la relación inicial de los alumnos con las tareas que se les proponen. Como ya hemos anunciado, la gestión colectiva del conocimiento en la clase también plantea interrogantes e incertidumbres con relación a la inclusión educativa. Al respecto, se discute sobre dos cuestiones estrechamente relacionadas: 
una vinculada al progreso en el nivel de elaboración de los procedimientos que movilizan los niños y otra, a las decisiones a tomar cuando se comparten en el espacio público del aula las producciones que realizaron individualmente o en pequeños grupos.

Orientada por su preocupación de hacer avanzar los procedimientos de los niños para problemas aditivos, la maestra de tercero clasifica las producciones recogidas para la indagación diferenciando entre aquellas basadas en el conteo -que fueron mayoritarias- de las que se apoyan en cálculos con descomposiciones decimales de los términos:

M 3: O sea, mi desafío es ahora volver, decir "bueno, ¿cómo podemos hacerlo sin contar para atrás? ¿Cómo lo podríamos hacer sin usar la grilla?”. Este es mi desafío en este momento.

La cuestión del progreso en los procedimientos de los alumnos, abierta en este contexto de indagación por el planteo de esta maestra, abarcó dos planos en las discusiones sostenidas: el de los avances dentro de un mismo grado y el que implica considerarlos a lo largo de los distintos años escolares.

Con relación al interrogante de la maestra que acabamos de citar-que se ubica en el primero de los planos mencionados-, algunas integrantes del EC van volcando diferentes propuestas: aumentar el rango de los números para obstaculizar el conteo, promover que la grilla sea soporte para contar de a 10 y no solo de a 1 y relacionar ambas estrategias, utilizar el recurso del dinero (billetes de \$10) como nexo entre el conteo y la descomposición decimal y confrontar distintos procedimientos en instancias de trabajo colectivo de la clase. Todas estas ideas -si bien no se desarrollaron en ese momento- aportan potencialmente elementos para la elaboración de intervenciones y, sobre todo, de explicaciones por parte de las docentes para promover el abandono del conteo a favor del cálculo, toda vez que se expone ante los alumnos una red de relaciones que permite establecer puentes entre ambas estrategias. Sin embargo, la confrontación colectiva entre distintos procedimientos propuestos por los niños es problemática y había sido tematizada previamente: hay una tensión entre alentar la difusión de procedimientos más elaborados y cuidar que todos los alumnos los puedan comprender Sadovsky et al., (2019).

La directora advierte, en reiteradas oportunidades, cerca de la necesidad de prestar mucha atención al modo de gestionar en el espacio colectivo de la clase la diversidad de producciones, en particular cuáles se seleccionan, cuidando 
que no queden alumnos fuera de la discusión. Este señalamiento supone, para nuestro equipo de investigación, un Ilamado a revisar la hipótesis según la cual el análisis colectivo de estrategias avanzadas contribuye al progreso de toda la clase en tanto puede constituir un modo de conectar a los niños con otras posibilidades. Sabemos, sin embargo, que en la práctica este impacto es incierto -puede o no ocurrir según las relaciones que cada alumno establezca con su propia producción- y que las decisiones que al respecto tomen los docentes se realizan en el marco de esa incertidumbre. Al conversar sobre nuestra hipótesis con la directora, ella la considera válida como perspectiva de trabajo pero, advierte sobre la necesidad de examinar, cada vez, el contexto de la clase. Más específicamente, señala que es prioritario tomar en cuenta el posicionamiento de los niños que se muestran más alejados del trabajo matemático.

Se mencionó un segundo plano en el análisis de los progresos de las estrategias que ponen en juego los alumnos, el del avance de grado a grado. Cada docente explicita entonces las estrategias más utilizadas por los niños para los problemas de resta, así como el rango de números en el cual se plantean los problemas. A partir de esta "puesta en común" respecto de los focos que se hacen en cada año escolar, se plantea la necesidad de realizar acuerdos institucionales. Así lo expresa la maestra de tercer grado, cuya preocupación principal -recordemos- está ligada a lograr que los niños transformen sus procedimientos:

M 3: Entonces, llega un momento en que, bueno, quizás, podríamos hacer algo institucional que quede documentado para que podamos ir ahí a ver; porque es lo que me pasa a mí, (...) que propongo secuencias que me parece que funcionarán y es difícil a veces. Tener una trayectoria para los chicos.

Como consecuencia de las discusiones que surgen, se pone de manifiesto el carácter colectivo de la enseñanza -aquello que es posible con un grupo de alumnos está condicionado, en parte, por su experiencia anterior- y es a partir de esa evidencia que se propone documentar los acuerdos que se realicen. Estos acuerdos, que van surgiendo del análisis, no se limitan a listar una serie de estrategias que se distribuirían entre los grados, sino más bien se va formulando una trayectoria para los alumnos en términos de red de relaciones para ir desarrollando y retomando a través del tiempo.

Entendemos que toda esta propuesta de acuerdos es constitutiva de la construcción de un marco común, favorecida por la existencia del EC. Asimismo, la intención de documentarlo es un modo de fortalecer la memoria 
institucional y retoma a cargo de las maestras una práctica de registro instalada en el EC, cuyo valor se fue haciendo explícito en tanto referencia para volver sobre las discusiones.

Nos resulta interesante destacar que, la idea de trayectoria mencionada por la maestra de tercer grado, va teniendo una doble acepción a lo largo de las conversaciones: como proyección de la enseñanza y como recorrido que hacen los alumnos.

La idea de trayectoria va alternando entre una referencia a la historia escolar de un niño, una mirada sobre todo un grupo y una perspectiva de los grupos a lo largo de todos los grados, entramando conceptualmente los procesos de enseñanza y aprendizaje. Ya sea que se aluda a la enseñanza o al aprendizaje, a todo un grupo, a toda la escuela o a un niño, las reflexiones sostenidas mantienen en este tramo de la discusión un carácter general, que pone en segundo plano la inmediatez de las acciones del aula.

\subsection{CENTRACIÓN EN LOS RECURSOS PARA EL ANÁLISIS}

La necesidad de analizar y organizar los datos, que fueron elaborados a partir de preguntas específicas del grupo de maestras, favorece la construcción de herramientas de análisis y fortalece una posición interpretativa que contribuye a subrayar el carácter hipotético de la enseñanza. Comentaremos a continuación el modo en que se fueron reconstruyendo las ideas de los alumnos y, luego, nos referiremos al valor de producción que tuvo en el EC la exigencia de organizar la información.

a) ¿Qué me dice la hoja de los chicos?

La pregunta parece orientar un proceso de reconstrucción de las ideas de los alumnos, cuando las maestras se internan en el conjunto de hojas que los niños han entregado. Al querer interpretar las ideas puestas en juego en cada procedimiento, se pone de manifiesto la insuficiencia de considerar solo la traza escrita para comprenderlas.

Una primera cuestión que surge en las conversaciones es la consideración de gestos y actitudes de los niños durante las resoluciones para poder desentrañar con mayor precisión su proceso. Es así como importa si un niño borró qué, en qué momento-, si revisó y/o corrigió, si recurre a estrategias que oculta 
-esconde las manos para contar con los dedos- o si, por el contrario, agrega anotaciones que no utilizó. Veamos algunos ejemplos:

M 1: Richard hizo todos circulitos y, en vez de tacharlos, borró los 18. Los borró y volvió a contar uno por uno, todos los que quedaban. No se confundió en ninguno.

Ejemplos como este contribuyen a que se tome conciencia del valor de observar a los alumnos en el desarrollo de su trabajo para reconstruir un procedimiento. Esto confronta con una práctica usual en la que después de proponer la tarea a los niños, los docentes se retiran para retomar la interacción recién después de que han finalizado. Si bien se suele explicitar que esta práctica apunta a la autonomía de los alumnos y que la observación es un control que la perjudicaría, el ejemplo ayuda a matizar estos supuestos, toda vez que la observación se orienta a entender cómo piensan y sostenerlos en su tarea.

Una de las maestras destaca que aquello que se plasma en la hoja no espeja necesariamente el proceso de resolución usado por el niño:

M 3: Ves, ahí tenés un caso. Francisco contó para atrás y después anotó la cuenta parada, como un agregado. O sea, no siempre la cuenta responde al procedimiento.

En este caso, la traza de la hoja muestra algo muy distinto de lo que el alumno hizo, lo cual da lugar a dos reflexiones en el EC: reconoce allí un problema de resta y puede resolverlo contando pero, a la vez, desestima su procedimiento tal vez porque considera que no es del todo legítimo y que la escritura de la cuenta tiene más valor.

Las revisiones sobre la propia producción que realizan los niños también se convierten en datos relevantes a considerar:

M $6^{\circ}$ : Algunos me dieron las hojas, al rato viene uno y me dice "ime das la hoja? Porque me parece que en algo me confundi".

¿Qué agrega esta información a la interpretación que se va construyendo? Entendemos que "habla" de un posicionamiento de compromiso intelectual con la tarea por parte del alumno y, en estrecha relación, muestra un rasgo de autonomía en tanto vuelve reflexivamente sobre la acción realizada para modificarla. A partir del relato de la maestra y de la intervención de la investigadora, el grupo analiza la resolución inicial del alumno y su corrección tratando de 
interpretar, en términos de conocimientos matemáticos, los cambios introducidos. Se produce en el EC un juego entre el hecho de saber que el niño se quedó pensando después de haber entregado su hoja y la necesidad de entender las relaciones aritméticas involucradas en su accionar.

En reiteradas oportunidades las maestras relatan que solicitan a los alumnos que no borren los intentos que van haciendo. Esta intervención se relaciona con el propósito de resguardar elementos relevantes para la reconstrucción del trabajo de los niños, a la vez que se les comunica el valor de los intentos, de las búsquedas, de los procesos, además de los resultados. En una dirección similar a que los niños y la clase en su conjunto legitimen -y confíen en- los recursos utilizados, se ubica el pedido de que no oculten los dedos cuando los usan para contar.

Las hojas de los niños las condujeron a considerar otros datos relativos al trabajo matemático que apoyaran las interpretaciones. Es así como traen a colación-además de lo relevado en el momento en que resuelven- informaciones más generales que las llevan, por ejemplo, a despreocuparse por un error que entienden como una distracción o también a tener cierta cautela cuando se habla de un alumno de otro grado que no conocen. Complementan además el análisis referencias al recorrido tanto del grupo como de cada niño.

b) La interacción entre maestras e investigadoras a propósito de la clasificación de los procedimientos de los niños

¿Cómo organizar los datos recogidos alejándose de las tradicionales categorías correcto-incorrecto (o cualquiera de sus variantes)? ¿Por qué habría que hacerIo? Esta cuestión llegó a la mesa del EC de la mano de una de las maestras que trajo los trabajos de su grado ya agrupados según una clasificación que a ella misma le resultaba insuficiente.

M 4: El criterio de clasificación fue difícil [...] Veintiuno aplicaron el algoritmo. De esos, ocho tienen errores menores, así que hay un porcentaje alto que hizo bien. Cinco hicieron una variedad de cosas que son muy dificiles de clasificar, difíciles de entender, esas serían las más interesantes de analizar...son historias distintas, yo las separé.

Al referirse a historias diferentes para las producciones incorrectas, la docente subraya la insuficiencia de su categorización para comprenderlas. Incluso duda de cuánto comprenden algunos niños cuyas resoluciones son correctas. Sostiene también que la variable tiempo juega un rol importante en las resoluciones 
y no es capturada en ese modo de organizar los datos. La maestra se encuentra insatisfecha por cómo había organizado el material ya que -reconoce- no le permite comprender los conocimientos que acompañan las producciones de los alumnos. Las investigadoras recogen esta preocupación y la incluyen en la síntesis que abre la reunión siguiente:

Como siempre, meternos en el análisis de las producciones de los chicos nos entusiasma y eso volvió a ocurrir en la reunión pasada. [...]. M $4^{\circ}$ planteó que clasificó las estrategias de los chicos pero que no le resultó fácil. Nunca es fácil porque el criterio de clasificación es personal y está muy orientado por lo que queremos saber, por lo que nos parece que debería suceder y por el rumbo que pensamos que habría que tomar. De manera que una segunda tarea que proponemos es la discusión de criterios de clasificación y la sistematización de las producciones de cada grado. Por supuesto que la clasificación se entrelaza con el análisis, por eso proponemos una tarea que sea "un poco y un poco" en la que, a medida que analizamos, construyamos criterios de clasificación.

Al recuperar las preocupaciones de la maestra, las investigadoras aportan elementos relativos al proceso de clasificación, propios del campo de la investigación: los criterios, lejos de ser preexistentes a la actividad de organización de los datos, se construyen en función de los problemas que se busca estudiar en una dialéctica entre clasificación y análisis. Esta intervención resuena en las maestras que, tras su lectura se preguntan sobre el significado de los criterios de clasificación, habilitando la posibilidad de profundizar la comprensión de esta práctica.

M 4: Pero, ¿̇a qué llamamos criterios de clasificación?

${ }^{111}$ : Podemos referenciarnos en lo que vos hiciste cuando el otro día trajiste tu relevamiento ordenado de los datos. Vos hiciste una clasificación. Además, dijiste que te había resultado difícil, cosa que nosotros compartimos. Porque el criterio no es algo ajeno a uno, no está afuera y lo agarro... cuando uno clasifica lo hace en función de algo, en base a algo.

La investigadora hace referencia al trabajo de la maestra como punto de apoyo para seguir reflexionando sobre los modos de organizar los datos y, en ese movimiento, le atribuye la capacidad de producir ella misma criterios.

\footnotetext{
11 Investigadora
} 
Este proceso de estudio de los datos de las aulas, al favorecer la producción de estrategias para la acción docente así como enriquecer la capacidad de análisis de todas las participantes del EC, sobrepasa ampliamente la producción de un diagnóstico que parecía ser la propuesta inicial (queremos saber dónde estamos parados, conocer la realidad). La búsqueda de una foto estática que intentaba capturar un momento fue cobrando, al calor del análisis, un relieve y un dinamismo que fueron dando cuenta de la cantidad de vericuetos que enfrentan los docentes para sostener la intención de incluir a todos los alumnos en el proyecto educativo.

\section{LA ESCRITURA COMO PROYECTO COMPARTIDO ENTRE DOCENTES E INVESTIGADORES: UN ESPACIO PARA PROFUNDIZAR EL SENTIDO DE LAS ACCIONES REALIZADAS}

Una vez desarrollada una primera vuelta de análisis, el equipo de investigación evalúa la necesidad de focalizar y profundizar las ideas que se fueron abriendo. Venía siendo una preocupación nuestra, de cara a los objetivos de la investigación, atrapar los núcleos de las conversaciones que se sostenían en el EC, ya que muchas veces se dispersaban tocando una infinidad de temas y quedaba la impresión que era difícil saldar en términos conceptuales las elaboraciones que iban surgiendo. Tomando en cuenta que las docentes venían planteando la necesidad de documentar el trabajo realizado como base para reformular juntas el proyecto de enseñanza de la matemática en la escuela, las investigadoras proponen una escritura conjunta para sistematizar los resultados de la indagación. Esta escritura surge entonces de la confluencia entre la necesidad de la escuela de conservar lo realizado y proyectar líneas de acción a futuro y la del equipo de investigación, de profundizar las discusiones y dar tiempo a conceptualizarlas. Se reúnen de esta manera, tras un objetivo común, un interés más ligado al funcionamiento institucional, con uno que busca estrategias metodológicas para promover el avance en la producción de conocimiento matemático-didáctico en el EC (Bednarz, 2013).

Compartimos el tramo de la reunión en el que las investigadoras invitan a las maestras a escribir los propósitos de la indagación y en el que se expresa la convergencia de intereses en esta escritura: 
I: O sea, una primera cosa que nosotras proponemos a ustedes, si están de acuerdo, es recuperar juntas y escribir con más detalle, qué propósito teníamos. Para qué hicimos ese relevamiento de procedimientos. Pensábamos hacer un texto juntas, ponerlo ahí [proyectarlo con el cañón a medida que se va escribiendo] para que lo vayamos viendo, corrigiendo y que quede como referencia institucional para el futuro, ustedes decían eso. Entonces, vamos escribiendo y corrigiendo juntas.

M $4^{\circ}$ : Claro, no solo un registro hacia atrás de lo que uno va aplicando, sino para llegar a acuerdos a futuro, de cuáles deberíamos compartir, me parece, ¿no?

El planteo de escritura conjunta genera entusiasmo y da lugar a una escena compleja, con numerosas idas y vueltas en la que cada término o idea que se va a escribir genera una discusión que va mucho más allá del significado estricto de aquello que se quiere expresar. Al revisar la reunión, las investigadoras que la coordinaron dan cuenta de una gran tensión entre sostener el proyecto de escritura y habilitar los debates que suscitaba el hecho de volver sobre las motivaciones de la indagación. El desorden al que refieren las coordinadoras -y que reencontramos al leer el registro- parece ser un rasgo de la exigencia de producción que esta situación supone. Había una preocupación constante de las maestras por ser fiel a las intenciones que las comandaron, llevando a prolongar las discusiones en la búsqueda de mayor minuciosidad. Además, la tarea que se encara es novedosa para todas: por una parte, es la primera vez que en el EC se vuelve con cierta rigurosidad sobre un proyecto que abarcó un tiempo relativamente prolongado para precisar los propósitos que lo orientaron; por otra, la escritura conjunta, desarrollada en el ámbito escolar, con tiempos limitados para arribar a una cierta producción, acentúa la complejidad mencionada.

Compartimos a continuación un análisis de la producción que tuvo lugar en el marco de la complejidad que acabamos de describir, refiriendo a: los temas de discusión que estuvieron presentes y que se profundizaron a partir de nuestra convocatoria a escribir los propósitos de la indagación; las reflexiones que suscita la necesidad de elegir los términos para expresar una idea; y las consideraciones que se desarrollan en el EC sobre el alcance y los límites de la indagación. 


\subsection{RELACIONES ENTRE ENSEÑANZA Y APRENDIZAJE EN UN MARCO INSTITUCIONAL}

La pregunta de una de las investigadoras dirigida a comenzar el proceso de escritura lleva a ampliar las ideas que se habían expresado cuando se propuso el proyecto. Recordemos que, en un primer momento, las maestras habían pensado que disponer de producciones de todos los niños sobre un mismo problema permitiría tener un panorama sobre las estrategias de los alumnos, así como conocer cuántas se mostraban muy distantes respecto de sus expectativas. Esto se expresó en aquella oportunidad como conocer la realidad, saber dónde estamos paradas. La investigadora retoma estas expresiones y convoca a expandirlas:

I: ¿Para qué hicimos eso? Una primera idea que apareció es ver dónde estamos parados, conocer la realidad. Pero, ¿̇le podemos poner un poco más de letra a esto? Decirnos a nosotras con un poco más de detalle cuál era el propósito, para qué hicimos este relevamiento de procedimientos. Eso nos ayudaría a entender más nuestras preocupaciones, nuestras preguntas, las satisfacciones, las insatisfacciones, y eso va a permitir armar conclusiones.

M $4^{\circ}$ : Porque hay que conocer la dificultad para poder abordarla, si no si uno no conoce dónde están nuestras dificultades, cuando digo "nuestras" hablo de la institución, pero hablo de nuestras dificultades como docentes y nuestras dificultades en el manejo de los contenidos en los alumnos.

De la intervención anterior, surgen tres ideas: se necesita conocer para poder actuar; los problemas son de la enseñanza, atañen tanto a docentes como a alumnos toda vez que los maestros buscan comprender las dificultades de los niños con los contenidos; y se asumen por parte del colectivo docente, ubicándose entonces en un contexto institucional.

Las relaciones entre las producciones de los alumnos y las estrategias docentes -que en la conversación exceden muchas veces la referencia a la indagación- son retomadas una y otra vez con diferentes matices. En un primer momento, pareciera considerarse el desempeño de los alumnos como reflejo estricto de la acción docente, apreciación que el desarrollo del análisis fue problematizando. Destaquemos que, en esa perspectiva, tal vez algo lineal, por una parte, las maestras asumen la responsabilidad sobre los aprendizajes de los niños en oposición a quienes justamente disocian enseñanza de aprendizaje pero, por otra, se podría correr el riesgo de leer en términos de culpabilización del docente la distancia de algunos niños frente a las tareas. Sabemos que 
ambos componentes están presentes en esta relación (Terigi, 2009). En nuestro caso, en tanto la conversación no se desliga casi en ningún momento de la referencia al marco institucional, queda más atenuada la tendencia a cuestionar la acción individual de cada maestra. Como grupo coordinador, nos interesó problematizar las relaciones entre enseñanza y aprendizaje impulsando intencionalmente hacia una visión más dialéctica.

M 4: [leyendo lo que había quedado escrito en el texto] Conocer la realidad de nuestros alumnos como producto de nuestras estrategias.

I: ¿Es un reflejo súper transparente?, ¿es tan absoluto? Pregunto. Lo que el pibe hace, ¿es el fiel reflejo del trabajo del maestro? Estoy problematizándolo. Porque pareciera que, si yo miro al pibe, directamente miro al maestro.

M $4^{\circ}$ : Lo que yo hice y lo que hicieron todas las anteriores. No miro a la maestra solamente en ese instante, miro toda la trayectoria. Es ahí donde nosotras podemos plantearnos los errores, los aciertos.

La pregunta de la investigadora abre a que se expliciten matices de la relación entre aprendizaje y enseñanza: la responsabilidad se atribuye al colectivo docente y, a través de él, alcanza a cada maestra. Asimismo, se sostiene que el trabajo de los alumnos siempre está enmarcado institucionalmente, aun en los casos en los que los procedimientos comportan una gran originalidad. En este sentido, se relativiza una idea bastante difundida según la cual existiría un accionar puramente espontáneo por parte de los niños: Lo que hacen siempre está vinculado con lo que alguien les enseñó, aunque no seamos nosotras, no es espontáneo. Enseñamos descomposiciones y a partir de ahí ellos hacen. A los argumentos que nutren las relaciones entre enseñanza y aprendizaje, se suma otro señalamiento: los niños hacen en función de lo que piensan que se espera de ellos y esto puede motorizar -cuando son capaces de usar de manera autónoma recursos que las maestras han alentado- u obstaculizar-cuando, en el afán de actuar conforme a lo que suponen como expectativa hacia ellos, se inhiben de poner en juego otras estrategias más propias, tal vez menos elaboradas- (Brousseau, 1980, 2007).

La mirada hacia los alumnos que no logran un desempeño autónomo en el trabajo matemático del aula hace visible la insuficiencia de estrategias de intervención únicas y, en consecuencia, la necesidad de transformarlas para provocar interacciones específicas que entren más en diálogo con el pensamiento de cada uno. Interesa, así lo explicita la maestra de cuarto grado, entender 
cómo se manifiesta el conocimiento en acción, es decir, frente a las situaciones que enfrentan los niños. Se plantea entonces la necesidad de incluir estas búsquedas en las prácticas docentes y, a su vez, la de comprender por qué algunas interacciones con ciertos niños no han tenido los resultados esperados. Las maestras incluyen estas reflexiones como propósitos de la indagación, aun sabiendo que fueron elaborados en el contexto del análisis posterior a la realización.

En síntesis, las relaciones entre enseñanza y aprendizaje quedan enmarcadas en un proyecto institucional de largo plazo en el que van interviniendo las distintas maestras; la pregunta por los saberes de los alumnos es también la pregunta por las expectativas y valoraciones de las docentes y sus estrategias. Los esfuerzos de algunos niños por poner en juego ciertos procedimientos hablan de aquello que se quiso transmitir y a la vez la distancia que a veces presentan con relación a las tareas conduce a interrogantes e hipótesis específicas -sobre lo que "no funcionó", sobre lo que podría ser fructífero-.

\subsection{LA ELECCIÓN DE LOS TÉRMINOS PARA LA ESCRITURA, FUENTE DE REVISIONES Y DE NUEVAS RELACIONES}

Son numerosos los autores que han descripto el papel productor que pueden tener las situaciones de escritura cuando la reflexión sobre el lenguaje promueve transformaciones en las ideas que se quieren comunicar (Scardamalia y Bereiter, 1992). Al verse confrontadas a la necesidad de elaborar un texto sobre los propósitos de la indagación, las maestras revisan muchos términos que son usuales en las conversaciones entre docentes cuando refieren tanto a las posibilidades de sus alumnos como a las alternativas de su quehacer. Es así que expresiones como "Ios alumnos no podían resolver", "Ios chicos con dificultad", "procedimientos inadecuados" o "buenas estrategias", entre otras, son puestas en cuestión cuando quedan plasmadas en el texto que se está elaborando. Lejos de centrarse en una preocupación formal, se trata de objeciones que son el resultado de una incomodidad: la de cargar con significados casi únicos, muy instalados en las prácticas docentes, ciertos hechos relativos a los intercambios del aula que el análisis realizado en el EC Ilevó a considerar críticamente.

Al proyectar en la pantalla el texto que se iba elaborando, se facilitó una revisión casi inmediata de la escritura. Compartimos a continuación distintos tramos en los que las reflexiones sobre la escritura vuelven sobre los sentidos en juego. 
No sé si "buenas" es la palabra

I: [Leyendo de la pantalla] Conocer la realidad de nuestros alumnos como producto de nuestras estrategias.

M 3: Mantener las buenas y buscar otras que vamos a tener que descubrir, las que nos dieron resultado, mantenerlas.

M 4ㅇ: No sé si "buenas" es la palabra, la verdad.

M $3^{\circ}$ : Mantener las que resultan...

M 4: Las más eficientes, las más... cuando digo "eficiente" quiero decir "lo que esperamos".

Pareciera que el adjetivo "buenas" no expresa la relación entre las expectativas docentes y los procedimientos de los chicos. Entendemos que la objeción del término se vincula con la necesidad de explicitar que una estrategia no se considera en sí misma sino por la función que tiene -o no- de promover en los alumnos la movilización de conocimientos relativos a la intención docente. Este adjetivo tampoco se acepta para referirse a los trabajos de los alumnos. Efectivamente, cuando las maestras revisan críticamente la atracción que les provocaban ciertas estrategias muy elaboradas y la poca atención que les habían prestado a los niños que se mostraban más ajenos al trabajo del aula, vuelven sobre este término.

M 4: Tuvimos hasta el momento la mirada de los lindos procedimientos..., ¿̇qué pusiste? ... bba a decir alguna cualidad...

M $3^{\circ}$ : Lo de enriquecer...

M $4^{\circ}$ : No, los que nos llamaban la atención, eso queremos decir. Porque cuando D dijo que estábamos encandiladas con esos procedimientos sin prestar atención a las hojas en blanco, recién ahí me hizo un clic y me dije "es verdad".

M 4: Bueno, sí. Que nos asombraban de alguna manera, la realidad es que nos quedábamos asombradas.

M 2: Claro, decíamos "mirá cómo hizo esto, ¿cómo lo pensó?".

M $4^{\circ}$ : Sobre aquellos procedimientos más elaborados y que nos asombraban, en todo caso.

M $2^{\circ}$ y $4^{\circ}:$ Y sacá "lindos procedimientos".

De manera similar al ejemplo anterior, "lindos" no resulta adecuado para precisar las razones por las que ciertos procedimientos habían ocupado un considerable tiempo de análisis en el EC. Las maestras evocan el interés que les 
suscitaba comprender las relaciones involucradas en algunas producciones de los niños, al tiempo que señalan que ese asombro les opacó la mirada hacia los que parecían no poder.

\section{"Lograban" es más esperanzador}

I: Acá están las ideas, ahora necesitaríamos darle forma y armar un texto para que nos quede documentado. ¿Quieren que vayamos leyendo? [Lee] La intención tuvo que ver con los que no podían resolver...

M 4: El "podían" lo podríamos cambiar por "lograban". "Poder" es medio terminante. "Lograban" es más esperanzador. De aquellos niños que no lograban resolver... aún, que no lograban aún resolver las situaciones presentadas.

La incomodidad alude en este tramo a un modo categórico de considerar a los alumnos y a sus posibilidades con relación al aprendizaje. La expresión "no lograban aún" subraya una posición provisoria del alumno que incorpora la modificación de esa situación en el futuro. La calificación de "esperanzador" releva una dimensión afectiva vinculada a la confianza tanto en el alumno (va a lograr resolver) como en la docente (vamos a lograr que resuelva).

No puedo saber si los chicos tienen una dificultad

Al referirse en el texto a los niños que motivaron la propuesta de la indagación, el término "dificultad" se pone en cuestión. Queremos subrayar que, en las conversaciones entre docentes, suele ser un modo de nombrarlos. Entendemos que algo del análisis realizado junto con la exigencia que impone la escritura lleva a buscar un modo más ajustado para describirlos.

M $4^{\circ}$ : Punto y aparte, como otra cosa, para qué lo hicimos. Para saber qué estrategias usan los chicos...

M $2^{\circ}$ : Con dificultades.

M $3^{\circ}$ : Yo pondría: para saber qué estrategias usan los chicos con dificultad...en realidad, yo no puedo saber si los chicos tienen una dificultad..., sería los chicos que no pudieron resolver en base a las estrategias que nosotros les enseñamos... No sabemos si tienen una dificultad... Pareciera que la dificultad la tiene el pibe, cuando por ahí, quizás la dificultad fue nuestra porque no le ofrecimos la estrategia... 
La idea que se impugna es que la dificultad es una condición del alumno al tiempo que se asume el fenómeno como una posible manifestación de la relación didáctica. Se abren así interrogantes sobre las prácticas de enseñanza y se sugiere la necesidad de seguir explorando. Asimismo, se expresa un cuidado por evitar una clasificación de los alumnos que podría derivar en su estigmatización.

Una mirada de conjunto sobre estas revisiones permite apreciar que las maestras se hacen responsables del significado de las ideas que se quieren comunicar, asumiendo una posición de autoría en el contexto de sus prácticas, novedosa para ellas. Se posibilita así una desnaturalización de ciertos términos a los que se suele apelar en las conversaciones entre docentes sin detenerse a examinar el alcance de los significados que portan.

\subsection{EL ALCANCE Y LOS LÍMITES DE LA INDAGACIÓN DESDE LA PERSPECTIVA DE LAS MAESTRAS}

Los análisis realizados, las idas y vueltas, cada una con nuevos matices y precisiones, en cierto momento, llevan al grupo a detenerse en sus reales alcances. Toda la conversación hizo visible que, para saber qué le pasa a un pibe, en el sentido de comprender qué ideas moviliza frente a las situaciones, cuáles son las razones por las que no llega a establecer relaciones pertinentes entre los datos del problema y la pregunta que se formula, entender por qué se muestra distante pero también acceder a las ideas que sí puede movilizar, es necesario sostener interacciones específicas que vayan a la búsqueda de estas respuestas. En ese marco, se cuestiona el alcance de aquello que puede conocerse a partir de interpretar una única producción que el alumno realizó en forma escrita y sin interacción con la maestra.

La indagación se plantea con el propósito explícito de entender mejor la posición de los niños distantes que dejan la hoja en blanco. Su puesta en marcha da lugar a un análisis que excede por mucho aquello que era posible interpretar solo a partir de las producciones puntuales. Se reconoce entonces la necesidad de realizar otras intervenciones para acceder a aquello que los alumnos saben. El recorrido realizado a raíz de la indagación permite identificar sus límites. Esto constituye desde nuestro punto de vista un avance en dos direcciones: una, en la afirmación de una posición investigativa que implica cuidar a lo largo del recorrido las relaciones entre los interrogantes que se buscan 
responder y las acciones que se realizan para lograrlo y, otra, en una comprensión más profunda de los requerimientos que presenta la enseñanza cuando se propone la inclusión de todos los niños.

\section{CONCLUSIONES}

En este artículo hemos dado cuenta del proceso de producción desarrollado en el espacio de trabajo colaborativo de una escuela a partir de la realización de una indagación sobre las producciones de los alumnos de todos los grados, planteada por una de las maestras. Esta iniciativa surge como respuesta a la necesidad de disponer de estrategias para interactuar con aquellos niños que se mostraban más distantes del trabajo matemático en las aulas, explicitada inicialmente por la directora y reconocida por el conjunto de las docentes. La realización de una acción específica y colectiva, que se proponía la búsqueda de respuestas sobre aspectos que inquietaban al conjunto, otorgó al proyecto - así lo entendemos- un carácter investigativo e institucional que fue central para el involucramiento de todas las docentes.

A medida que se iban examinando las producciones de los alumnos en el espacio colaborativo, se iban elaborando posibles estrategias de intervención docente que, miradas globalmente, fueron constituyendo un repertorio más atento a las particularidades de cada niño y, en consecuencia, más fundamentado. En este sentido, el avance del análisis fue dando lugar a la construcción de respuestas parciales para el problema identificado. Asimismo, el trabajo de reconstrucción de las ideas de los niños a partir de sus producciones contribuyó a fortalecer una posición exploratoria y a tomar conciencia de la necesidad de observar todo el proceso de producción (y no solo su estado final) así como de recurrir a otros elementos de contexto para alcanzar una interpretación más ajustada. En ese marco, el proceso de clasificación de los procedimientos como herramienta interna a la indagación se pudo diferenciar de las acciones evaluativas y de sus categorías clásicas -correcto e incorrecto- que, en esta oportunidad, mostraron sus límites para dar respuesta a las preguntas que las maestras se hacían. También se hizo presente en los intercambios el requerimiento de hacer acuerdos entre las diferentes maestras que tomaran en cuenta una progresión entre los grados con relación a los procedimientos puestos en juego por los alumnos, sin que se perdieran de vista las trayectorias personales. 
El proceso de análisis realizado a partir de las producciones obtenidas en el marco de la indagación profundiza y amplía los propósitos que inicialmente se habían planteado. Las primeras inquietudes, conocer la realidad, saber dónde estamos paradas, parecían referirse a los desempeños de los alumnos, determinados por la enseñanza. Sin embargo, tanto el avance del análisis como la situación de escritura habilitaron nuevas -y más dialécticas- relaciones entre enseñanza y aprendizaje.

Por una parte, se subraya que los alumnos actúan en un marco institucional que genera expectativas sobre ellos a las que tratan de responder, lo cual condiciona el abanico de posibilidades que se permiten desplegar. Aun cuando los niños desarrollan procedimientos originales en los que toman decisiones y eligen qué recursos poner en juego, estos están encuadrados en estrategias generales que se han enseñado. Asimismo, se señala que los alumnos construyen una representación de lo que se espera de ellos como resultado de todo su recorrido escolar y se valoriza así la necesidad de acuerdos institucionales. Estos se conciben en términos de trayectorias de enseñanza que se internan más minuciosamente en las relaciones implicadas en los conocimientos que los niños tienen que aprender, que no son usualmente capturadas en los típicos listados de contenidos en los que se distribuyen temas entre los diferentes grados.

El ámbito de discusión generado favorece la movilización de la experiencia acumulada en la escuela para dar cuenta de que los posicionamientos de los niños, sobre todo de aquellos que muestran distancia con relación a las tareas matemáticas, pueden ser transformados si existe una fuerte intencionalidad pedagógico-didáctica orientada a tal fin. La hoja en blanco requiere ser interpretada en la interacción sostenida con los niños que será insumo para la elaboración de estrategias de intervención.

Mirando retrospectivamente este proceso, surge una última reflexión de corte metodológico. Una maestra propuso este proyecto y el grupo se lanzó a su realización sin haberse detenido a examinar previamente de manera exhaustiva ni sus propósitos ni sus alcances. Como investigadoras, nos "encontramos" con esta propuesta en una reunión y decidimos habilitar su desarrollo en el marco del espacio colaborativo pensando que, cualquiera fuera su devenir, constituiría una situación de aprendizaje para todas. Entendemos que si la hubiéramos frenado para lograr que se precisaran sus propósitos de manera previa, hubiéramos entorpecido la iniciativa de las maestras que tal vez no tenían en el momento inicial todas las anticipaciones que requiere la formulación de un proyecto de carácter investigativo. Esas "anticipaciones" se fueron elaborando "a 
posteriori" en el transcurso del trabajo en el que, con los papeles de los niños arriba de la mesa, se reconstruían una y otra vez los propósitos y los sentidos y se proyectaban acciones futuras. En el marco de confianza ya construido en este espacio y, basadas en los resultados obtenidos, entendemos que dar cabida a una propuesta en la que no estaba tan clara para nosotras la relación entre el problema que se estaba considerando y la estrategia que se proponía para esclarecerlo, contribuyó a que las docentes asumieran una posición productora, tal como se describe en este trabajo. Un pequeño paso en la comprensión de las condiciones para una genuina colaboración.

\section{REFERENCIAS}

Baquero, R. y Terigi, F. (1996). En búsqueda de una unidad de análisis del aprendizaje escolar. Apuntes. http://www.bibliopsi.org/docs/carreras/obligatorias/CFP/educacional/chardon/1\%20primer\%20cuatri\%202020/P\%20 1.3\%20En\%20Busqueda\%20de\%20una\%20Unidad\%20de\%20Analisis\%20-\%20Baquero.pdf

Bednarz, N. (2013). Regarder ensemble autrement: ancrage et développement des recherches collaboratives en éducation au Québec. En N. Bednarz, Recherche collaborative et pratique enseignante. Regarder ensemble autrement (pp. 13-29). L'Harmattan.

Bednarz, N. y Proulx, J. (2010). Développement professionelle des enseignants en mathématiques. En B. De Lièvre, A. Braun, V. Carelle, y W. Lahaye, Éducation et formation. Travail en communautés, collaboration et partenairiats pour le dévéloppement professionelle des enseignants, e-293 (pp. 21-36). Université de Mons.

Brousseau, G. (1980). L'échec et le contrat. Recherches, 41, 177-182. https://hal. archives-ouvertes.fr/file/index/docid/483149/filename/Brousseau_1980_ echec_et_contrat.pdf

Brousseau, G. (2007). Iniciación al estudio de la teoría de las situaciones didácticas. Libros del Zorzal.

Douady, R. (1984). Jeux de cadres et dialectique outil-objet dans lénseoignement des mathématiques. Une réalisation dans tout le cursus primaire. Thèse de doctorat d'état. Université Paris VII. https://tel.archives-ouvertes.fr/tel01250665/ 
Peltier-Barbier, M.-L. (2004). Dur d'enseigner en ZEP. Analyse des pratiques de professeurs des écoles enseignant les mathématiques en réseaux d'éducation prioritaire. La Pensée Sauvage.

Proulx, J. (2013). Réflexions épistémologiques sur la recherche collaborative en didactique: possibilités et excès. En N. Bednarz, Recherche collaborative et pratique enseignante. Regarder ensemble autrement (pp. 327-349). L'Harmattan.

Robert, A. (2003). De l'idéal didactique aux déroulements réels en classe de mathématiques: le didactiquement correct, un enjeu de la formation des (futurs) enseignants (en collège et lycée). (I. N. Pédagogique, Ed. Didaskalia (22).

Robert, A., y Rogalski, J. (2002). Le système complexe et coherent des pratiques des enseignants de mathématiques: une double approche. La revue canadienne de l'enseignement des sciences des mathématiques et des technologies, 2(4), 505-528.

Sadovsky, P., Itzcovich, H., Becerril, M., Quaranta, M., y García, P. (2019). Trabajo colaborativo entre docentes e investigadores en didáctica de la matemática: de la reflexión sobre las prácticas a la elaboración de ejes de análisis para la enseñanza. Educación Matemática, 31(2), 105-131.

Sadovsky, P., Itzcovich, H., Quaranta, M., Becerril, M., y García, P. (2016). Tensiones y desafíos en la construcción de un trabajo colaborativo entre docentes e investigadores en didáctica de la matemática. Educación Matemática, 28(3), 9-29.

Sadovsky, P., Quaranta, M. E., García, P., Becerril, M., y Itzcovich, H. (2019). Trabajo colaborativo entre docentes e ivnestigadores en didáctica de la matemática. Reflexiones metodológicas. Revista digital Contextos de Educación, (26), 41-49.

Sadovsky, Quaranta, Itzcovich, Becerril, y García. (2015). Producción matemático-didáctica: una experiencia de planificación colaborativa entre maestros e investigadores. En A. Pereyra, y D. Fridman, Prácticas Pedagógicas y Políticas Educativas. Investigaciones en el territorio bonaerense (pp. 221-250). Editorial Universitaria.

Scardamalia, M., y Bereiter, C. (1992). Dos modelos explicativos del proceso de composición escrita. Infancia y Aprendizaje, 15(58), 43-64.

Sensevy, G. (2011). Le sens du savoir. Éléments pour une théorie de l'action conjointe en didactique. De Boeck. 
María Emilia Quaranta, María Mónica Becerril, Patricia García, María Paula Pérez, Patricia Sadovsky

Terigi, F. (2009). El fracaso escolar desde una perspectiva psicoeducativa. Hacia una mirada situacional. Revista iberoamericana de educación, 23-39. https://doi.org/10.35362/rie500659

MARÍA EMILIA QUARANTA

Domicilio: Coronel Toscano 569. Villa Sarmiento.

Provincia de Buenos Aires, Argentina

C.P. 1706

Teléfono: 5491150585913 